University of Windsor

Scholarship at UWindsor

$1-21-2020$

\title{
Transverse lipid organization dictates bending fluctuations in model plasma membranes
}

\author{
Brett W. Rickeard \\ University of Windsor \\ Michael H.L. Nguyen \\ University of Windsor \\ Mitchell Dipasquale \\ University of Windsor \\ Caesar G. Yip \\ University of Windsor \\ Hamilton Baker \\ University of Windsor
}

See next page for additional authors

Follow this and additional works at: https://scholar.uwindsor.ca/chemistrybiochemistrypub

Part of the Biochemistry, Biophysics, and Structural Biology Commons, and the Chemistry Commons

\section{Recommended Citation}

Rickeard, Brett W.; Nguyen, Michael H.L.; Dipasquale, Mitchell; Yip, Caesar G.; Baker, Hamilton; Heberle, Frederick A.; Zuo, Xiaobing; Kelley, Elizabeth G.; Nagao, Michihiro; and Marquardt, Drew. (2020).

Transverse lipid organization dictates bending fluctuations in model plasma membranes. Nanoscale, 12 (3), 1438-1447.

https://scholar.uwindsor.ca/chemistrybiochemistrypub/151

This Article is brought to you for free and open access by the Department of Chemistry and Biochemistry at Scholarship at UWindsor. It has been accepted for inclusion in Chemistry and Biochemistry Publications by an authorized administrator of Scholarship at UWindsor. For more information, please contact scholarship@uwindsor.ca. 


\section{Authors}

Brett W. Rickeard, Michael H.L. Nguyen, Mitchell Dipasquale, Caesar G. Yip, Hamilton Baker, Frederick A. Heberle, Xiaobing Zuo, Elizabeth G. Kelley, Michihiro Nagao, and Drew Marquardt 


\title{
Journal Name
}

\section{ARTICLE TYPE}

Cite this: DOI: $00.0000 / x x x x x x x x x x$

\section{Transverse lipid organization dictates bending fluctuations in model plasma membranes}

\author{
Brett W. Rickeard ${ }^{\ddagger a}$ Michael H.L. Nguyen, ${ }^{\ddagger a}$, Mitchell DiPasquale, ${ }^{\ddagger a}$ Caesar G. Yip, ${ }^{a}$ \\ Hamilton Baker, ${ }^{a}$ Frederick A. Heberle, ${ }^{b, c}$ Xiaobing Zuo, ${ }^{d}$ Elizabeth G. Kelley, ${ }^{e}$ Michihiro \\ Nagao, ${ }^{e, f}$ and Drew Marquardt ${ }^{* a, g}$
}

Received Date

Accepted Date

DOI: $00.0000 / x x x x x x x x x x$

\begin{abstract}
Membrane undulations play a vital role in many biological processes, including the regulation of membrane protein activity. The asymmetric lipid composition of most biological membranes complicates theoretical description of these bending fluctuations, yet experimental data that would inform any such a theory is scarce. Here, we used neutron spin-echo (NSE) spectroscopy to measure the bending fluctuations of large unilamellar vesicles (LUV) having an asymmetric transbilayer distribution of high- and low-melting lipids. The asymmetric vesicles were prepared using cyclodextrin-mediated lipid exchange, and were composed of an outer leaflet enriched in egg sphingomyelin (ESM) and an inner leaflet enriched in 1-palmitoyl-2-oleoyl-phosphoethanolamine (POPE), which have main transition temperatures of $37^{\circ} \mathrm{C}$ and $25^{\circ} \mathrm{C}$, respectively. The overall membrane bending rigidity was measured at three temperatures: $15^{\circ} \mathrm{C}$, where both lipids are in a gel state; $45^{\circ} \mathrm{C}$, where both lipids are in a fluid state; and $30^{\circ} \mathrm{C}$, where there is gel-fluid co-existence. Remarkably, the dynamics for the fluid asymmetric LUVs (aLUVs) at $30^{\circ} \mathrm{C}$ and $45{ }^{\circ} \mathrm{C}$ do not follow trends predicted by their symmetric counterparts. At $30^{\circ} \mathrm{C}$, compositional asymmetry suppressed the bending fluctuations, with the asymmetric bilayer exhibiting a larger bending modulus than that of symmetric bilayers corresponding to either the outer or inner leaflet. We conclude that the compositional asymmetry and leaflet coupling influence the internal dissipation within the bilayer and result in membrane properties that cannot be directly predicted from corresponding symmetric bilayers.
\end{abstract}

\section{Introduction}

Eukaryotic plasma membranes (PM) are characterized by an asymmetric distribution of lipids between the exoplasmic and cytosolic leaflets of the bilayer. ${ }^{1+3}$ This compositional distinction has evolved such that high-melting and uncharged lipids predominantly comprise the outer leaflet, while low-melting and nega-

\footnotetext{
a Department of Chemistry and Biochemistry, University of Windsor, Windsor, ON, Canada

${ }^{b}$ University of Texas Health Science Center, Houston, TX, USA

${ }^{c}$ The Bredesen Center for Interdisciplinary Research and Graduate Education, University of Tennessee, Knoxville, TN, USA

${ }^{d}$ X-ray Science Division, Argonne National Laboratory, Lemont, IL, USA

e NIST Center for Neutron Research, National Institute of Standards and Technology, Gaithersburg, MD, USA

${ }^{f}$ Center for Exploration of Energy and Matter, Department of Physics, Indiana University, Bloomington, IN, USA

${ }^{g}$ Department of Physics, University of Windsor, Windsor, ON, Canada.

\$ These authors contributed equally to this work

* Corresponding author email: drew.marquardt@uwindsor.ca

$\dagger$ Electronic Supplementary Information (ESI) available: [details of any supplementary information available should be included here]. See DOI: 00.0000/00000000.
}

tively charged lipids form most of the inner leaflet. Specifically, healthy mammalian cells sequester nearly all of the high-melting sphingomyelin (SM) in the outer leaflet of the PM, while phosphatidylethanolamine (PE) and negatively charged lipids are almost completely segregated into the inner leaflet. ${ }^{45}$ Consequently, this lipid composition difference contributes to large differences in the physicochemical properties of the two layers. Despite the ubiquity of membrane asymmetry in living cells, there is still much to be learned about the importance of this lipid organization and how it impacts the structure, dynamics, and ultimately the function of the PM.

Most biologically occurring lipids intrinsically form spherical soft bilayers whereby elastic constants can be assigned and used to characterize the mechanical properties of the membrane. For example, the bending modulus $\kappa$ quantifies the membrane rigidity, which governs the membrane fluctuations on the nanoscale 6 6 and has profound biological relevance. Thermally-induced membrane undulations influence numerous biological processes including the interactions and insertions of membrane proteins ${ }^{8-11}$, the size and morphology of lipid rafts ${ }^{12}-14$, and transport vesicle 
formation 15 , 17 , to name but a few.

The near ubiquity of asymmetric membranes in nature implies that symmetric bilayers are not adequate for characterizing PM properties. Still, the vast majority of research into membrane mechanical properties has made use of symmetric model membranes, in part owing to the experimental challenges posed by the preparation of asymmetric vesicles. Moreover, asymmetry contributes additional dynamic processes such as transverse lipid diffusion (flip-flop) that can couple to mechanical properties and thereby complicate the analysis of experimental data. 18 Recent theoretical efforts examined the effects of asymmetry on the membrane dynamic properties including area compressibility modulus and membrane viscosity, and found that lipid asymmetry couples to the dynamic modes and relaxation times. 19 However, within this theoretical framework, the dispersion relations for an asymmetry in bending modulus were deemed non-trivial and thus not reported. Experimental measurements of bending rigidity in asymmetric bilayers will better inform such theoretical treatments, and may provide information about the strength of interleaflet coupling, i.e. the effects that the two leaflets impose on one another. To this end, we investigated the effects of compositional asymmetry on the bending modulus of model asymmetric plasma membranes using neutron spin-echo (NSE). NSE directly measures the effective bending modulus, which contains the effects of internal bilayer dissipation, and is the only technique capable of resolving these effects. These internal dissipations are likely highly sensitive to the differences in leaflet properties present in asymmetric bilayers, but this correlation still requires elucidation and thus necessitates the present study.

The asymmetric composition of the mammalian PM suggests that its two leaflets will have different mechanical properties. In symmetric model membranes, ordered lipids such as those found in the PM outer leaflet typically form more rigid bilayers, while the more disordered lipids found in the PM inner leaflet tend to make softer bilayers 20 . How these differences manifest in an asymmetric bilayer depends on the extent of coupling between the leaflets with respect to their differing physicochemical and mechanical properties (termed interleaflet coupling), and possibly other factors. This area of research is largely unexplored. Our experimental strategy was to prepare model membranes that mimic the ordered/disordered lipid asymmetry of a natural PM and measure its membrane structure and mechanical properties. The strength of interleaflet coupling can then be inferred by comparing these properties with symmetric bilayers of the corresponding inner and outer leaflet compositions.

\section{Results}

Asymmetric large unilamellar vesicles (aLUVs) $\approx 200 \mathrm{~nm}$ in diameter were prepared by introducing ESM into the outer leaflet of POPE vesicles using cyclodextrin-mediated lipid exchange.21.24 As ESM, and not POPE, possesses a choline group, these aLUVs can be interrogated with NMR to determine the transbilayer lipid distribution, as described below. Diminished bilayer integrity promotes lipid flip-flop 25 , which can reduce the asymmetry and potentially modify membrane bending dynamics. To ensure vesicle integrity we rigorously monitored leaflet composition at differ- ent stages of sample preparation and data collection. Small-angle neutron scattering (SANS) measurements showed a vesicle form factor at low scattering wave vector transfer, Q, values for both the acceptor POPE vesicles and final aLUVs (Fig. S2), indicating that neither sample preparation conditions nor trace amounts of residual cyclodextrin altered the vesicle structure. To augment the SANS measurements, we used dynamic light scattering (DLS) to periodically check the vesicle size, and found no significant changes during aLUV preparation. All symmetric LUV controls were subjected to the same wash steps and $\mathrm{D}_{2} \mathrm{O}$ exchanges as the aLUV.

Asymmetric lipid distributions in aLUVs were quantified from solution ${ }^{1} \mathrm{H}$-NMR spectra measured in the presence of $\mathrm{Pr}^{3+}$, an extravesicular paramagnetic lanthanide ion. Externally added $\mathrm{Pr}^{3+}$ does not permeate into the vesicle interior on the timescale of the NMR measurement ${ }^{21 / 26}$ and therefore interacts only with outer leaflet lipid headgroups to induce a downfield shift of the ESM choline resonance. We note that the ethanolamine of POPE does not contribute to the ${ }^{1} \mathrm{H}-\mathrm{NMR}$ signal ${ }^{24}$. The observed choline signal is a superposition of shifted and unshifted resonances whose relative areas are proportional to the amount of ESM in the outer and inner leaflets, respectively (Fig. 1) 26 . Immediately prior to SANS and NSE measurements, aLUVs whose outer leaflets were enriched in ESM (i.e., $\mathrm{POPE}^{\text {in }} / \mathrm{ESM}^{\text {out }}$ ), exhibited an unequal area ratio (Fig. 113). Further, the lipid distribution in symmetric vesicles was assessed using ${ }^{1} \mathrm{H}$-NMR to demonstrate that the spontaneous molecular curvature differences between ESM and POPE does not generate spontaneous compositional asymmetry.

Fig. 11p shows a ${ }^{31} \mathrm{P}-\mathrm{NMR}$ spectrum which corresponds to the overall distribution of ESM and POPE in the aLUVs. The mol fractions of ESM and POPE were determined to be 0.25 and 0.75 , respectively. Combining results from the ${ }^{1} \mathrm{H}-\mathrm{NMR}$ and ${ }^{31} \mathrm{P}-\mathrm{NMR}$ spectra resulted in the overall lipid distribution of the vesicular bilayers. The aLUV outer leaflet contained an ESM mole fraction of $0.44\left(\chi_{E S M}=0.44\right)$ indicating partial replacement of outer leaflet POPE, with a small amount of ESM $\left(\chi_{E S M}=0.06\right)$ translocating to the inner leaflet during the exchange step (Table S1). The cartoon illustration of the aLUV composition is shown in Fig. 1C. This composition remained stable for the duration of the NSE measurements as determined by ${ }^{1} \mathrm{H}-\mathrm{NMR}$, suggesting little to no flip-flop over the course of 3 days. The observed temporal stability of ESM asymmetry is consistent with a recent report of slow flipflop in both isotopically 25 and chemically 2427 asymmetric vesicles.

The asymmetric bilayer structure was determined as a function of temperature by modeling small-angle neutron and X-ray scattering data. $\frac{2324}{2}$ A typical example of the data, fit and X-ray electron density profile are shown in Fig. 1P and 1E. Scattering profiles were well fit by a model in which ESM is enriched in the outer leaflet and POPE in the inner leaflet and did not change over the course of 24 hours, providing additional evidence for aLUV stability. Bilayer structural parameters, for both LUVs and aLUVs, are summarized in Tables S2 and S3. We note that structural parameters of the symmetric LUVs follow the expected temperature and composition dependence. For example, the bilayer thickness $\left(D_{B}\right)$ decreases with increasing temperatures and $D_{B}$ increases 

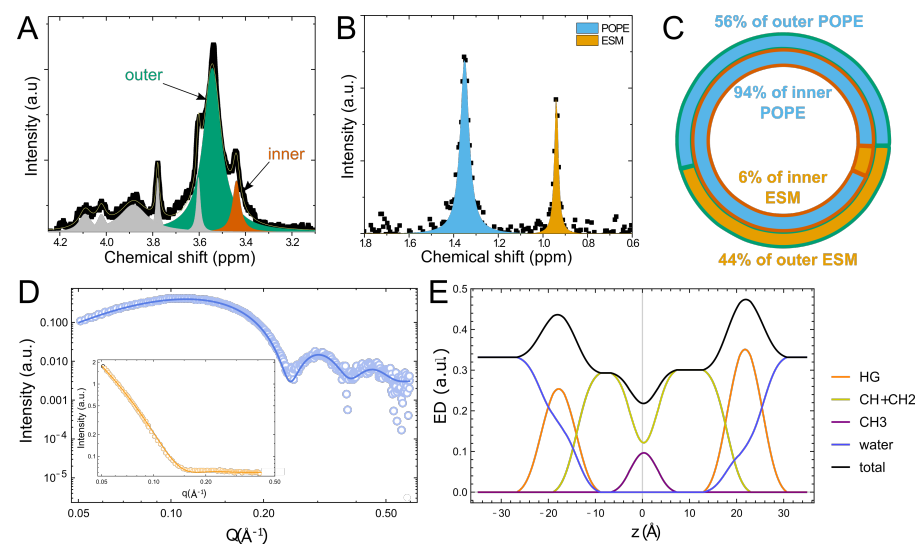

Fig. 1 A) ${ }^{1} \mathrm{H}$-NMR of aLUV in the presence of the shift reagent $\mathrm{Pr}^{3+}$. The red peak represents the protected choline groups on the inner leaflet, the green peak is the outer leaflet choline groups, and the grey peaks arise from other groups on the lipid and residual CD peaks (3.6 and $3.8 \mathrm{ppm}$ ). B) ${ }^{31} \mathrm{P}-\mathrm{NMR}$ of the aLUV to determine the ratio of ESM (orange) and POPE (blue) present. C) Cartoon representation of the aLUV generated. The blue presents POPE lipids, and orange represents ESM, highlighting their distribution. D) SAXS (main) and SANS (inset) data of the aLUV at $30{ }^{\circ} \mathrm{C}$ are represented by circles, and the jointly optimized model fits are represented by solid lines. E) The resultant electron density (ED) profile calculated from the optimized models from $D$. The black curve is the total ED which is the sum of the terminal methyl groups of the hydrocarbon chains $\left(\mathrm{CH}_{3}\right)$, the bulk of the hydrocarbon $\left(\mathrm{CH}+\mathrm{CH}_{2}\right)$, the headgroup $(\mathrm{HG})$ and the water.

with ESM content.

NSE was used to measure the bending dynamics of aLUVs as well as a set of symmetric binary ESM/POPE mixtures at various temperatures, allowing us to observe changes in the membrane stiffness with respect to the bilayer composition and temperature. NSE is well suited for measuring collective dynamic properties of lipid bilayers compared to other techniques (Fig. 2 top). The normalized intermediate scattering function $I(Q, t) / I(Q, 0)$ vs. Fourier time for aLUVs at $30{ }^{\circ} \mathrm{C}$ is shown in Figure 2. The measured dynamics for the aLUVs follow the same scaling seen in the symmetric vesicles in this work and those reported in literature, $728-30$ supporting that the same bending fluctuations are being measured in the aLUVs as those in symmetric models. The data follow a stretched exponential as predicted by Zilman and Granek for membrane bending fluctuations based on Helfrich's model that treats the membrane as a thin elastic sheet,

$$
\frac{I(Q, t)}{I(Q, 0)} \simeq e^{-\left(\Gamma_{Z G} t\right)^{2 / 3}} .
$$

with the fits shown as the solid lines. $\frac{31}{31}$ The corresponding decay rates, $\Gamma_{Z G}$, follow the expected $Q^{3}$-dependence shown in the inset in Figure 2 with a slope that is inversely related to the effective bending modulus $\tilde{\kappa}$ by,

$$
\frac{\Gamma_{Z G}}{Q^{3}}=0.025 \sqrt{\frac{k_{B} T}{\tilde{\kappa}}} \frac{k_{B} T}{\eta},
$$

where $\eta$ is the solvent viscosity, $T$ is the absolute temperature, and $k_{B}$ is the Boltzmann constant.

Watson and Brown extended the theory used to treat NSE data to account for the fact that the membrane is not a thin structureless sheet and instead has a finite thickness by incorporating the effects of internal dissipation within the bilayer. ${ }^{32 \mid 33}$ They showed that the effective bending modulus measured by NSE is related to the intrinsic bending modulus, $\kappa$, through

$$
\tilde{\kappa}=\kappa+2 d^{2} k_{m}
$$

where $d$ is the height of the neutral surface from the bilayer midplane and $k_{m}$ is the monolayer compressibility modulus. Accordingly, a stiffer membrane will have a smaller decay constant as the membrane fluctuations are slower.
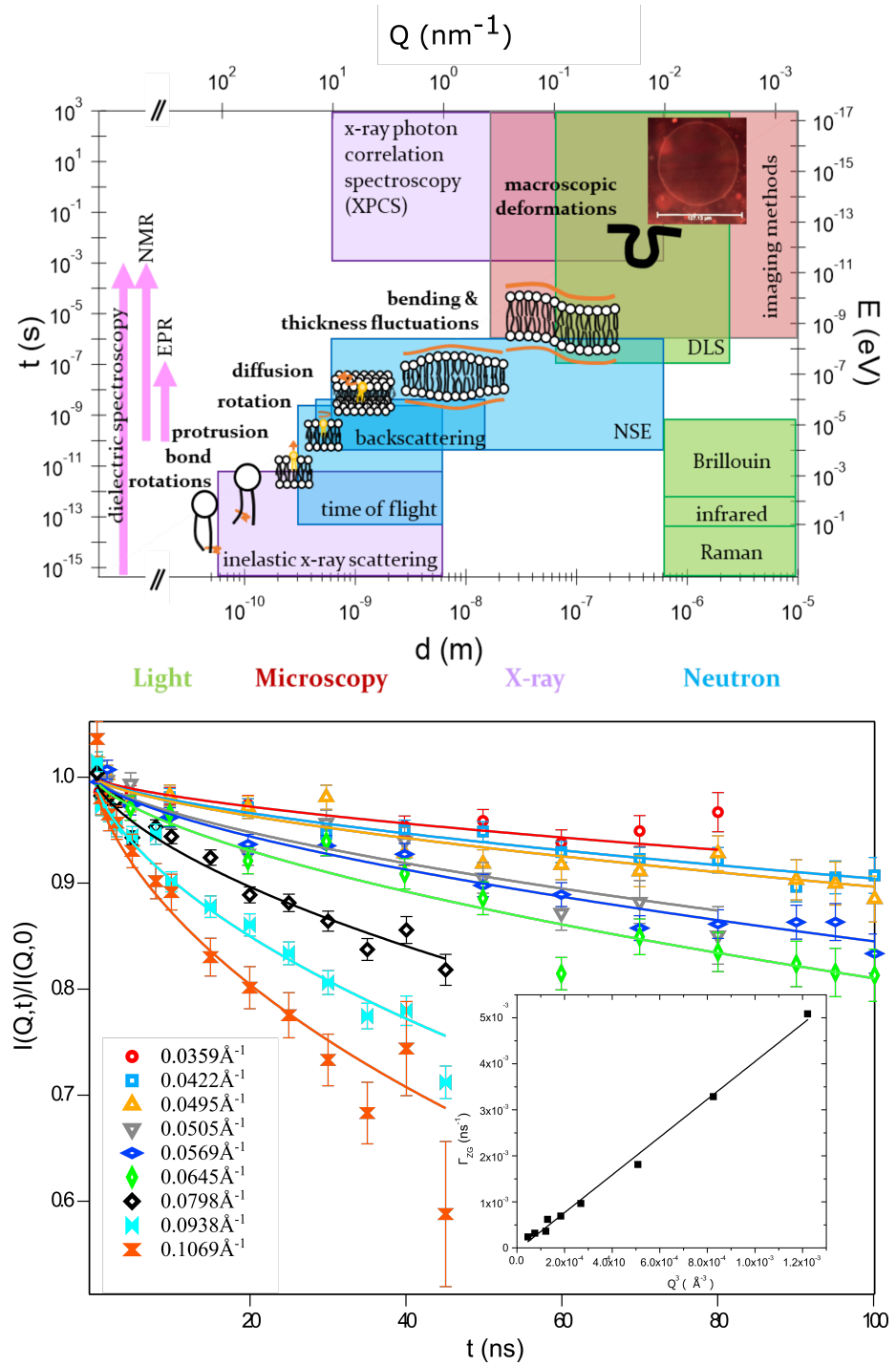

Fig. 2 Top Accessible length and time scales and corresponding energy and momentum transfer $(\mathrm{Q})$, for some spectroscopic techniques covering nanoscopic to macroscopic dynamics, covering a number of membrane dynamics. Figure adapted from ref $\left(\frac{34}{34}\right)$. BottomNormalized intermediate scattering function $I(Q, t) / I(Q, 0)$ measured by NSE for the aLUV in $\mathrm{D}_{2} \mathrm{O}$ at $30{ }^{\circ} \mathrm{C}$. The inset shows the linear dependence of the relaxation rate $\left(\Gamma_{Z G}\right)$ with respect to $Q^{3}$. Error bars represent one standard deviation here and throughout the manuscript.

Experimentally measured decay rates, normalized by $Q^{3}$, for symmetric and asymmetric membranes are plotted in Figure 3 a 
Table 1 Average composition $\left(\chi_{E S M}\right)$ and bending modulus $\kappa$ of asymmetric and symmetric bilayers composed of ESM and POPE.

\begin{tabular}{|c|c|c|c|c|}
\hline$\chi_{E S M}$ & sym/asym & $15^{\circ} \mathrm{C}$ & $\begin{array}{l}\kappa\left(k_{B} T\right) \\
30^{\circ} \mathrm{C}\end{array}$ & $45^{\circ} \mathrm{C}$ \\
\hline $0.0^{a}$ & sym & $433 \pm 51$ & $16.3 \pm 0.5$ & $10.4 \pm 0.2$ \\
\hline $0.25^{b}$ & sym & - & $22.2 \pm 0.7$ & $15.2 \pm 0.2$ \\
\hline $0.25^{b}$ & asym & $360 \pm 36$ & $61 \pm 2$ & $26.6 \pm 0.7$ \\
\hline $0.44^{c}$ & sym & $470 \pm 51$ & $48 \pm 2$ & $28.6 \pm 0.7$ \\
\hline 0.85 & sym & $638 \pm 77$ & $326 \pm 24$ & $32.9 \pm 0.9$ \\
\hline 1.0 & sym & $705 \pm 93$ & $492 \pm 45$ & $33 \pm 1$ \\
\hline \multicolumn{5}{|c|}{${ }^{a}$ POPE acceptor, $\approx$ aLUV inner leaflet composition } \\
\hline \multicolumn{5}{|c|}{${ }^{b}$ aLUV average composition } \\
\hline \multicolumn{5}{|c|}{${ }^{c}$ aLUV outer leaflet composition } \\
\hline
\end{tabular}

and show a clear temperature dependence, with the membranes becoming more dynamic as the temperature increases. The most dramatic change occurs at the transition from the gel to fluid phase from $15^{\circ} \mathrm{C}$ to $45^{\circ} \mathrm{C}$, with $\left\langle\Gamma_{Z G} / Q^{3}\right\rangle$ increasing by an order of magnitude in the fluid phase, where $\langle\ldots\rangle$ denotes averaging over all $Q$ values accessed. This significant increase in dynamics is consistent with the increased hydrocarbon chain disorder in the fluid phase (wherein the chains sample a larger conformation space), as well as an increase in lipid lateral and transverse diffusion, and the loss of long range positional order within the bilayer. $25|35| 39$ The order of magnitude increase in dynamics also agrees with the softening of the bilayers in the fluid phase and the order of magnitude decrease in bending modulus seen upon lipid melting. 40

Figure 3 also shows that the dynamics are relatively insensitive to composition at temperatures completely below ( $\square$ ) or above $(\boldsymbol{\Delta})$ the chain melting transitions of both POPE and ESM. The dynamics decrease modestly with increasing amounts of ESM at $45{ }^{\circ} \mathrm{C}$. In contrast, there is a strong composition dependence at the intermediate temperature of $30{ }^{\circ} \mathrm{C}(\bullet)$, with the average decay constant decreasing by nearly a factor of 10 with increasing ESM concentration. The sharp change in dynamics at high ESM concentrations indicates that these mixtures are no longer fluid and instead have either phase separated or condensed into an ordered (gel-like) phase, which is further supported by the comparatively high melting temperature of ESM at $37^{\circ} \mathrm{C}$. These observations are consistent with NSE analysis of other binary lipid mixtures that showed a sharp increase in bending modulus upon lipid phase separation 41/42. Meanwhile, the symmetric vesicles with low ESM concentrations $\left(\chi_{E S M}<0.5\right)$ and the aLUVs remain dynamic at the intermediate temperature of $30^{\circ} \mathrm{C}$.

Focusing on the biologically relevant fluid phase membranes reveals unique and unexpected behaviors for asymmetric membranes: the asymmetric vesicle dynamics bear no simple relationship to that of the symmetric membranes corresponding to the inner and outer leaflet compositions. Instead, the dynamics ap- pear to be an emergent property of the asymmetric transbilayer distribution of lipids that themselves have distinct melting transitions and order. The deviations in behavior are evident in the measured relaxation rates, as well as in the corresponding $\kappa$ values. Values for $\kappa$ can be calculated by substituting reported values for $d$ and $k_{m}$ in Eqns. 2 and 3 to give 7

$$
\frac{\Gamma_{Z G}}{Q^{3}}=0.0069 \sqrt{\frac{k_{B} T}{\kappa}} \frac{k_{B} T}{\eta} .
$$

Corresponding values of $\kappa$ calculated using Eqn. 4 are presented in Table 1 and plotted in Figure 33 . The $\kappa$ for the aLUVs at both temperatures are closer to the stiff outer leaflet, but are not a simple function of the individual leaflet properties (i.e., neither an arithmetic, geometric, or harmonic 43 average). Moreover, the anomalies are temperature-dependent: $\kappa$ for the aLUV falls between the leaflet values at $45^{\circ} \mathrm{C}$ while at $30^{\circ} \mathrm{C}$ the aLUVs are stiffer than both the outer and inner leaflets. The $\kappa$ measured for aLUVs at $30{ }^{\circ} \mathrm{C}$ is even more surprising given that the symmetric LUV with the outer leaflet composition has overall $75 \%$ more high-melting ESM, revealing that the asymmetry increases $\kappa$ and suppresses the bending fluctuations as illustrated in Figure $3 \mathrm{C}$.

\section{Discussion}

\subsection{Influence of lipid spontaneous curvature on bending rigid- ity}

It was recently reported that asymmetric giant unilamellar vesicles (GUVs) prepared by the phase transfer technique and composed of DOPC and POPC in the fluid phase also showed enhanced membrane rigidity as compared to their symmetric counterparts 18 , a result that was attributed to the different spontaneous curvatures $\left(J_{o}\right)$ of these lipids. ${ }^{44}$ While the results for ESM/POPE aLUVs at $30{ }^{\circ} \mathrm{C}$ are superficially consistent with this explanation, the $45{ }^{\circ} \mathrm{C}$ data, do not show the same dramatic difference in $\kappa$ between symmetric and asymmetric LUVs that was seen in the GUV study. It has been reported that the spontaneous curvatures of ESM and POPE exhibit a qualitatively different temperature dependence, wherein $J_{o}$ of ESM increases, and $J_{o}$ of POPE decreases, with an increase in temperature. $\frac{44}{4}$ As the spontaneous curvature difference between ESM and POPE monolayers increase as the temperature is raised, the effects of the spontaneous curvature should thus be more evident at the higher temperature. It is therefore unlikely that differences in the spontaneous curvature of ESM and POPE can account for the dampened bending fluctuations we observe in aLUVs at $30{ }^{\circ} \mathrm{C}$, but not at $45{ }^{\circ} \mathrm{C}$. Differences in lipid spontaneous curvature have also been addressed in previous structural measurements of aLUVs composed of POPE/POPC 24. In that study, the difference in spontaneous curvature between POPE and POPC could not explain the lack of interleaflet coupling, with respect to the area per lipid, in fluidphase POPE/POPC aLUVs. Taken together, a structural description of interleaflet coupling is insufficient in explaining the findings in this work. In general, lipid dynamics may be more sensitive to changes in composition and perhaps other system conditions than structural features, a conclusion in line with a previous finding where a dramatic change in lipid flip-flop and intervesic- 

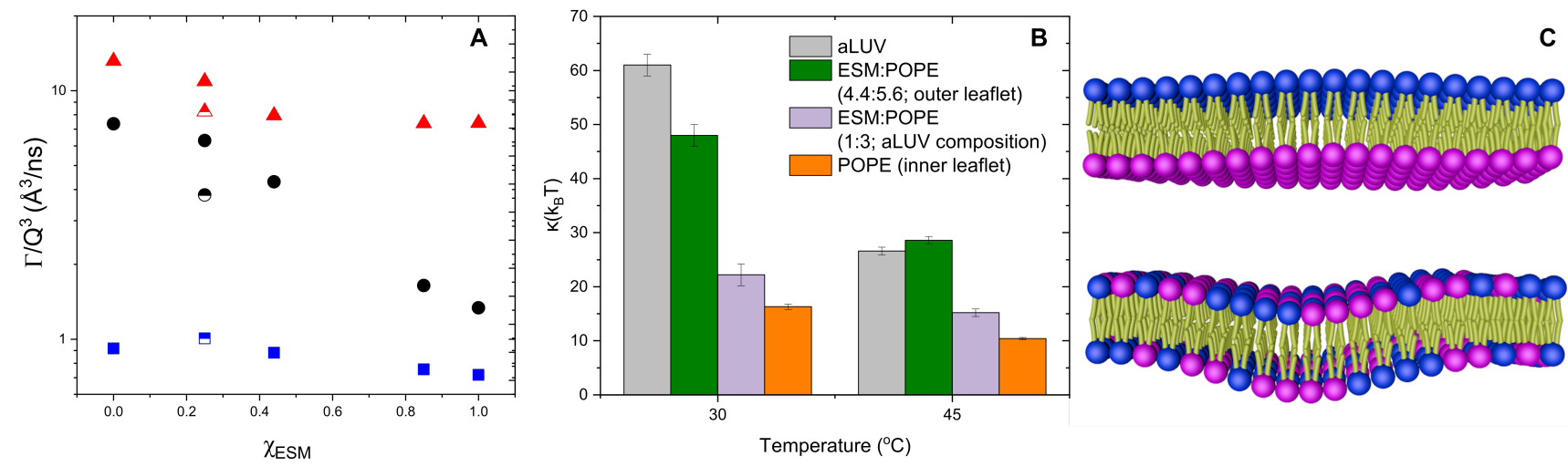

Fig. 3 Decay rate $\Gamma_{Z G}$ normalized by $Q^{3}$ averaged for all $Q$ for both aLUVs and LUVs as a function of $\left.\mathbf{A}\right) \mathrm{ESM}$ mole fraction $\left(\chi_{E S M}\right)$ at $15^{\circ} \mathrm{C}(\boldsymbol{\square}), 30^{\circ} \mathrm{C}$ $(\bullet)$ and $45^{\circ} \mathrm{C}(\boldsymbol{\Lambda})$. aLUV samples are indicated by half-filled points. B) Bending moduli $(\kappa)$ for the fluid aLUVs and symmetric vesicles with compositions corresponding to the inner leaflet, outer leaflet and overall aLUV composition measured with NSE (complete temperature series found in Fig. S4.C) Cartoon visualization of the observed bending rigidity for an asymmetric organization of ESM and POPE (top) and a symmetric mixture of ESM and POPE (bottom) at $30^{\circ} \mathrm{C}$.

ular exchange rates in the presence of methanol was observed before any structural deviations were detected by SANS, SAXS or DLS 45 .

Another important distinction of this work is that the dynamics were measured on the nanoscale with neutron spin echo and its results are uniquely sensitive to the internal dissipation dynamics within the membrane. Techniques such as flicker spectroscopy and micropipette aspiration probe the long wavelength dynamics that are slow compared to the motions of the lipids and have well-understood relationships to the intrinsic bending modulus. Consider the footprint of a membrane-bound protein compared to the overall size of the membrane itself; it is liken to the Burj Khalifa skyscraper being influenced by the curvature of the Earth. Thus, the relevance of microscopic bending fluctuation to a protein's structure-function is debatable. As mentioned above, NSE directly measures the effective bending modulus, that is the intrinsic bending modulus plus the effects of internal dissipation as suggested by Eq. 3 .

\subsection{Influence of bilayer phase state and lateral organization on bending rigidity}

The inability to recover asymmetric $\kappa$ values through a combination of the symmetric systems is not completely surprising. Examining the $\kappa$ values for the symmetric LUVs there is a clear $\chi_{E S M}$ dependence at $30{ }^{\circ} \mathrm{C}$ and $45{ }^{\circ} \mathrm{C}$ (at low $\chi_{E S M}$ ); however, the $\kappa$ values for the symmetric LUVs are not clear weighted averages of the pure lipid systems either. We have ruled out lateral phaseseparation of the outer leaflet as the driver for these observations. Because of the large increase in $A_{L}$ for aLUVs at $30{ }^{\circ} \mathrm{C}\left(54.2 \AA^{2}\right)$ from $15{ }^{\circ} \mathrm{C}\left(45.7 \AA^{2}\right)$, which corresponds closely to $A_{L}$ of the fluidphase LUVs (Table S2 and S3). From this, it is clear that the aLUVs are also fluid phase. Further, the presence of the faster dynamics indicates that these membranes are fully fluid at $30^{\circ} \mathrm{C}$. This observation is supported by the DSC thermogram of ESM/POPE aLUVs (Fig. S1) and agrees with previous NMR studies by Soni et al. that showed symmetric binary mixtures with a similar lipid compositions were disordered and fluid at $30{ }^{\circ} \mathrm{C} .46 / 477$ Further, wide angle X-ray scattering (WAXS) data demonstrate that mixtures composed of $\chi_{E S M}=0,0.25$ and 0.44 are fluid at $30^{\circ} \mathrm{C}$. The lack of a Bragg peak in these WAXS curves is indicative of no phase coexistence as well (Fig. S2).

The simplest, yet still plausible, explanation for the differences in the POPE/ESM mixed membranes dynamics (both LUVs and aLUVs) is the non-ideal mixing of POPE and ESM, even when both lipids are fluid. Past works have reported that PE and PC lipids de-mix based on differences in hydrogen bonding capa-

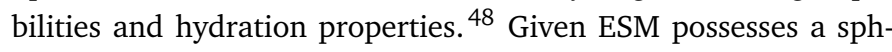
ingosine backbone and a choline headgroup, it seems reasonable to conclude the hydration and hydrogen bond differences are present in the ESM/POPE system. Furthermore, it has been demonstrated that low-melting PE lipids have minimal miscibility with high-melting PC lipids. ${ }^{49}$ Local inhomogeneities in the membrane composition due to non-ideal lipid mixing could indeed influence the dynamics at the length scale of the bilayer measured with NSE. $42 / 43$

\subsection{Vesicle and bilayer structure}

Although effective, the use of the phase transfer method to generate the asymmetric bilayers is a cause for concern. When working in an oil-rich environment, similar to those in the phase transfer set-up, there exists potential for oil entrapment within the vesicle bilayer, which can cause membrane defects and influence relevant bilayer properties, such as membrane thickness. Therefore, asymmetric bilayers constructed in the absence of hydrocarbon solvents, and where the bilayer structure has been intimately characterized, may constitute a more biologically faithful platform for these mechanical measurements.

Asymmetric vesicles generated by CD-mediated exchange were meticulously assessed for artifacts resulting from the preparation conditions. The analysis of SANS and SAXS data from isotopically asymmetric aLUVs showed that POPC and DPPC vesicles prepared by CD-mediated exchange had bilayer structures that were identical to those prepared by conventional vesicle preparation techniques. $2125 \mathrm{X}$-ray and neutron based techniques are 
particularly sensitive to the lipid interface and can reveal information at the bilayer surface, such as CD interactions. ${ }^{50 \mid 51}$ In the experiments presented here, both the initial POPE acceptor LUVs and the final aLUVs exhibit a spherical shell scattering form factor consistent with a bilayered vesicle, a clear indication that sample preparation conditions did not compromise the vesicle integrity. Moreover, the diameter of the aLUVs used in NSE experiments was $\approx 200 \mathrm{~nm}$, rendering the possibility of bilayer curvature or lipid packing effects unlikely. ${ }^{5}$ Further, we demonstrate that neither the bilayer curvature or the molecular curvature of the lipids cause spontaneous asymmetry.

Potential changes in the membrane structure and order do not explain the disparate temperature behavior of the aLUVs. The increase in $\kappa$ upon lowering the temperature from $45^{\circ} \mathrm{C}$ to $30^{\circ} \mathrm{C}$ in the symmetric ESM and ESM/POPE $\left(\chi_{E S M}=0.44\right)$ bilayers is consistent with the measured changes in the bilayer thickness (Table S3) according to the widely-used polymer brush model. ${ }^{[52}$ Structural measurements of the aLUVs indicate that the aLUV bilayer thickness changes only by $\approx 2 \%$ on cooling from $45^{\circ} \mathrm{C}$ to $30^{\circ} \mathrm{C}$, which cannot account for the nearly $200 \%$ change in $\kappa$, ruling out that the aLUV dynamics are explained solely by changes in the bilayer thickness.

\subsection{Mechanical properties of asymmetric vesicles}

The emergent behavior seen in the aLUV dynamics cannot be explained by mechanisms commonly seen in symmetric bilayers such as changes in membrane phase state, lateral organization, thickness or spontaneous curvature discussed above. Instead, we speculate that combining leaflets with unique chemical and physical properties into an asymmetric membrane influences the bending fluctuations measured on the nanoscale with NSE. The fluctuations at these short length and time scales are strongly influenced by not only the bending modulus, but also the interleaflet friction.

The extended theory by Watson and Brown that relates the effective bending modulus $\tilde{\kappa}$ measured with NSE to the true bending modulus $\kappa$ incorporates Seifert-Langer theory to account for the leaflet density and interleaflet friction. $\frac{32 \mid 33}{1 n}$ other words, the lipids do not redistribute between the leaflets quickly enough, leading to an effectively larger bending rigidity at the nanoscale. The effects of such leaflet density fluctuations have been confirmed in various symmetric lipid membranes, $7 / 28|30| 53$ but the asymmetric membranes studied here show dynamics that cannot be accounted for by this simple explanation. One likely possibility is that the asymmetric leaflet compositions and densities are influencing the internal dissipation within the bilayer. Alternatively, Eqn. 3 that links the true bending modulus $\kappa$ to the effective bending modulus $\tilde{\kappa}$, inherently assumes that the membrane is symmetric and depends on the definition of the neutral surface and the monolayer compressibility modulus.

The numerical pre-factor in Eqn. 4 used to calculate $\kappa$ assumes that the neutral surface, $d$, of each leaflet is at the interface between the lipid headgroup and hydrophobic tail. This assumption has worked well for other lipid systems, ${ }^{7 / 44}$ but the location of $d$ within the bilayer remains a topic of discussion even for sym- metric bilayers, and it is not obvious how $d$ would be defined in an asymmetric bilayer. Moreover, it is likely that the two leaflets of an asymmetric bilayer have different compressibility moduli which is not explicitly accounted for in the current model. It is also possible that the membrane asymmetry introduces other dissipation mechanisms or dynamic modes not yet considered theoretically. For example, asymmetry is predicted to induce a coupling between membrane thickness fluctuations and the movement of the membrane internal surface, which would lead to different relaxation behavior in an asymmetric and symmetric membranes. 19 These results indicate that the bending modulus of an asymmetric bilayer is not simply the sum of each monolayer bending modulus. This was also observed previously in studies of asymmetric GUVs. $\frac{18[54}{15}$ Therefore, the bending mechanisms of the leaflets could be different between symmetric and asymmetric ones, suggesting differences in interleaflet coupling and dissipation on a nanoscale.

\section{Conclusion}

To summarize, the data presented reveal that bending fluctuations in asymmetric membranes cannot be simply predicted from the properties of corresponding symmetric bilayers. We stress that the observations of anomalous dynamics in the aLUV are a true representation of the behavior, despite the current theoretical frameworks being unable to fully describe the mechanisms. Our data suggest that the stiffness of an asymmetric membrane is dictated by the more rigid leaflet, and that coupling of two leaflets with independent physicochemical properties introduces new contributions to the dissipation within the bilayer. The asymmetry-induced changes in the dynamics on the length-scale of the membrane itself are especially relevant to protein conformation changes and binding as well as intermembrane interactions that have been linked to the bending fluctuations. 55 These changes in rigidity undoubtedly have consequences for integral membrane proteins such as mechanosensitive pores, $58[59$ and likely impact processes such as endocytosis, exocytosis, and membrane trafficking. 60 Moreover, the strong interleaflet coupling observed here is entirely driven by lipids; indeed, lipids may be the major driving force for coupling in natural membranes, as peripheral proteins have not demonstrated such an ability. ${ }^{61}$ As membrane viscosity directly influences protein diffusion, and thus their ability to find interaction partners, these lipid-driven fluctuations may even be required for the proper function of some membrane proteins.

\section{Methods}

\subsection{Materials}

N-Acyl-4-sphingenyl-1-O-phosphorylcholine (egg sphingomyelin, egg SM), 1-palmitoyl-2-oleoyl-sn-glycero-3-phosphoethanolamine (16:0/18:1 PE, POPE) 1-palmitoyl-2-oleoyl-sn-glycero-3-phospho(1'-rac-glycerol) (sodium salt) [16:0/18:1 PG, POPG] were purchased from Avanti Polar Lipids (Alabaster, AL) and used as received (Fig. S5). Lipid stock solutions were prepared by dissolving the dry lipid powder in either HPLC-grade chloroform (ACP Chemicals Inc., Saint Lonard, QC) or in a combination of HPLC- 
grade chloroform and HPLC-grade methanol (Sigma-Aldrich, Oakville, ON). The unwanted sucrose and $\mathrm{m} \beta \mathrm{CD}$ was removed from the $\mathrm{ON})$ in a 2:1 ratio respectively. Methyl-beta-cyclodextrin $(\mathrm{m} \beta \mathrm{CD})$ was purchased from Sigma-Aldrich (Oakville, ON) and prepared as a $35 \mathrm{mmol} / \mathrm{L}(\mathrm{mM})$ stock solution in ultrapure $\mathrm{H}_{2} \mathrm{O}$. The sucrose was purchased from Sigma-Aldrich (Oakville, ON) and prepared as a $0.632 \mathrm{M}$ stock solution in ultrapure $\mathrm{H}_{2} \mathrm{O}$. Sodium chloride was purchased from Sigma-Aldrich (Oakville, ON) and prepared as a $20 \mathrm{mM}$ solution in $\mathrm{D}_{2} \mathrm{O}$. Praseodymium (III) nitrate hexahydrate $\left(\operatorname{Pr}\left(\mathrm{NO}_{3}\right)_{3} \cdot 6 \mathrm{H}_{2} \mathrm{O}\right)\left[\mathrm{Pr}^{3+}\right]$ was purchased from SigmaAldrich (Oakville, ON) and prepared as $40 \mathrm{mM}$ stock solution in $\mathrm{D}_{2} \mathrm{O}$. The deuterated chloroform $\left(\mathrm{CDCl}_{3}\right)$ was purchased from Cambridge Isotopes (Andover, MA). Ultrapure $\mathrm{H}_{2} \mathrm{O}$ was obtained from a Milli-Q purification system (Millipore-Sigma, Oakville, ON) and $99.9 \% \mathrm{D}_{2} \mathrm{O}$ was purchased from Cambridge Isotopes (Andover, MA).

\subsection{Preparation of Asymmetric Vesicles}

POPE (acceptor) and ESM (donor) lipid films were prepared by transferring the desired volumes of stock lipid solutions to separate glass scintillation vials. Bulk organic solvent was removed under gentle house vacuum and the resulting films were dried overnight $(\approx 12 \mathrm{~h})$ under vacuum at $50{ }^{\circ} \mathrm{C}$. POPE films were doped with a mole fraction of $5 \%$ POPG to help facilitate unilamellarity. Donor multilamellar vesicles (MLVs) were prepared by hydrating the ESM film with $0.632 \mathrm{M}$ sucrose solution to a lipid concentration of $20 \mathrm{mg} / \mathrm{mL}$. The film was preheated to 50 ${ }^{\circ} \mathrm{C}$ before hydration, followed by incubation at $50{ }^{\circ} \mathrm{C}$ with intermittent vortexing to fully disperse the lipids. The donor MLVs were then subjected to 3 freeze/thaw cycles at $-80{ }^{\circ} \mathrm{C}$ and $50{ }^{\circ} \mathrm{C}$ with intermittent vortexing. The donor MLVs were diluted 20fold with $\mathrm{H}_{2} \mathrm{O}$, immediately followed by centrifugation at 20,000 $\mathrm{x} \mathrm{g}$ for 30 minutes. The resulting supernatant was discarded, and the pellet was re-suspended with the $35 \mathrm{mM} \mathrm{m} \beta \mathrm{CD}$ solution to an 8:1 $\mathrm{m} \beta \mathrm{CD}$ to lipid ratio. The donor lipid $/ \mathrm{m} \beta \mathrm{CD}$ mixture was then incubated at room temperature for $2 \mathrm{~h}$ with mild stirring $(4 \mathrm{~Hz}$ ). Acceptor large unilamellar vesicles (LUVs) were prepared by hydrating the POPE film with the $20 \mathrm{mM} \mathrm{NaCl}$ solution to a lipid concentration of $10 \mathrm{mg} / \mathrm{mL}$. The subsequent MLVs were then subjected to 5 freeze/thaw cycles at $-80^{\circ} \mathrm{C}$ and 50 ${ }^{\circ} \mathrm{C}$ including vortexing after each thawing step. The acceptor LUVs were prepared by a Nanosizer AUTO Liposome Extruder ( $\mathrm{T} \& \mathrm{~T}$ Scientific, Knoxville, TN) equipped with $100 \mathrm{~nm}$ polycarbonate filter (T \& T Scientific, Knoxville, TN). The lipid suspension was passed through the filter 31 times at $45^{\circ} \mathrm{C}$. Asymmetric large unilamellar vesicles (aLUVs) were prepared by combining the acceptor LUVs with the donor/m $\beta \mathrm{CD}$ mixture at a ESM:POPE molar ratio of 2:1. Prior to bringing the donors and acceptors together, the donor $/ \mathrm{m} \beta \mathrm{CD}$ slurry temperature was raised to $35^{\circ} \mathrm{C}$. The donor/m $\beta \mathrm{CD} /$ acceptor mixture was then stirred gently (at $35{ }^{\circ} \mathrm{C}$ ) for 30 minutes, followed by an 8-fold dilution with $\mathrm{H}_{2} \mathrm{O}$ and centrifugation at 20,000 x $\mathrm{g}$ for 45 minutes. The supernatant was carefully removed as not to disturb the pellet. The supernatant was concentrated to $10 \mathrm{~mL}$ using an Amicon Stirred Cells (Millipore-Sigma, Oakville, ON) with a prewashed $100 \mathrm{kDa}$ Ultrafiltration Disc (Millipore-Sigma, Oakville, asymmetric LUVs via consecutive dilution/concentration cycles with $\mathrm{D}_{2} \mathrm{O}$ to a total $\mathrm{H}_{2} \mathrm{O} / \mathrm{D}_{2} \mathrm{O}$ dilution of $1000 \mathrm{x}$. On the aLUVs wash/concentration the volume was brought $\approx 6 \mathrm{~mL}$ of concentrated aLUVs.

\subsection{Determination of Bilayer Composition Via NMR}

The relative lipid composition of the aLUV was determined via ${ }^{31} \mathrm{P}-\mathrm{NMR}$. Water was removed from an aLUV aliquot using a CentriVap Benchtop Centrifugal Vacuum Concentrator (Labconco, Kansas City, MO) for $16 \mathrm{~h}$ at $50{ }^{\circ} \mathrm{C}$ to ensure all the water was removed. The resulting film was dissolved with $1 \mathrm{~mL}$ of deuterated chloroform $\left(\mathrm{CDCl}_{3}\right)$ to a lipid concentration of $\approx 1 \mathrm{mg} / \mathrm{mL}$. $700 \mu \mathrm{L}$ was loaded into an NMR tube for ${ }^{31} \mathrm{P}$-NMR measurement. ${ }^{31} \mathrm{P}$ NMR spectra were collected on an Avance III $500 \mathrm{MHz}$ spectrometer (Bruker, Billerica, MA) using Bruker TopSpin acquisition software, and analyzed with TopSpin 3.5. The observed chemical shifts were relative to the external standard for ${ }^{31} \mathrm{P}\left(85 \% \mathrm{H}_{3} \mathrm{PO}_{4}\right)$. The mole fraction $(\chi)$ of the POPE and ESM were determined by the intensity under the respective peaks. The relative distribution ESM between the aLUV leaflets was determined via ${ }^{1} \mathrm{H}$-NMR and the addition of a $\mathrm{Pr}^{3+}$ shift reagent as outline in Doktorova et al. ${ }^{22}$. ${ }^{1} \mathrm{H}$-NMR spectra were collected on an Avance III 600 MHz spectrometer (Bruker, Billerica, MA) using Bruker TopSpin acquisition software, and analyzed with TopSpin 3.5. The overall lipid organization of the aLUVs was calculated from the mole fractions of ESM and POPE determined from the integrated peak intensities of the lipid phosphates as measured by ${ }^{31}$ P-NMR and the distribution of ESM from ${ }^{1} \mathrm{H}$-NMR following the procedures for Heberle et al. ${ }^{21}$ and Doktorova et al. ${ }^{22}$. The composition of our aLUVs is summarized in Figure $1 \mathrm{C}$.

\subsection{Preparation of Symmetric Control Vesicles}

LUVs were prepared by transferring the desired volumes of stock lipid solutions to achieve the desired ratio. Bulk organic solvent was removed under a gentle stream of $\mathrm{N}_{2}$ and the resulting films were dried overnight $(\approx 12 \mathrm{~h})$ under vacuum at $50{ }^{\circ} \mathrm{C}$. All symmetric LUV films were doped with a mole fraction of $5 \%$ POPG to help facilitate unilamellarity. Films were hydrated with the $20 \mathrm{mM} \mathrm{NaCl}$ solution to a lipid concentration of $20 \mathrm{mg} / \mathrm{mL}$. One POPE control was hydrated with pure $\mathrm{D}_{2} \mathrm{O}$. The hydrated films were subjected to 5 freeze/thaw cycles at $-80{ }^{\circ} \mathrm{C}$ and 50 ${ }^{\circ} \mathrm{C}$ with intermittent vortexing after each thawing step. LUVs were generated by a Nanosizer AUTO Liposome Extruder (T \& T Scientific, Knoxville, TN) equipped with $100 \mathrm{~nm}$ polycarbonate filter ( $\mathrm{T} \& \mathrm{~T}$ Scientific, Knoxville, TN). The vesicle suspensions were passed through the filter 31 times at $45^{\circ} \mathrm{C}$. LUVs were then subjected to the same $\mathrm{D}_{2} \mathrm{O}$ washing/concentration steps as the aLUVs. The LUV controls were: POPE, POPE (hydrated with pure $\mathrm{D}_{2} \mathrm{O}$ ), ESM:POPE (1:1), ESM:POPE (4:1) and ESM.

\subsection{Neutron Spin-Echo (NSE) Spectroscopy}

Our data was taken on the spectrometer located on the NG-A guide at the NIST Center for Neutron Research (NCNR; Gaithersburg, MD) ${ }^{62}$. The momentum transfer, $Q$, ranged from $0.04 \AA^{-1}$ 
to $0.11 \AA^{-1}$. The wavelengths used in this experiment were $8 \AA$, allowing the higher region of the scattering vector to be reached, and $11 \AA$, which was chosen to access the lower momentum transfer regions at the expenditure of reduced beam intensity. These experimental settings allowed for Fourier times of up to $100 \mathrm{~ns}$, corresponding to bilayer motions on length scales of $\approx 0.1 \mathrm{~nm}$ to $10 \mathrm{~nm}$ and time scales of $0.1 \mathrm{~ns}$ to $100 \mathrm{~ns}$. The cells used for the NSE measurements had a path length of $4 \mathrm{~mm}$. Temperature was controlled within $0.5{ }^{\circ} \mathrm{C}$ and the samples were allowed to equilibrate the desired temperature for at least 30 minutes prior to starting the measurement. The subsequent NSE data was reduced using Data Analysis and Visualization Environment (DAVE) software package 63 .

\subsection{SANS and SAXS Measurements}

SANS measurements were conducted on the NGB 30m SANS instrument located at the National Institute of Standards and Technology Center for Neutron Research (NCNR, Gaithersburg, MD) 64 . Wavelengths of $6 \AA$ and $8 \AA$ were used, as well as three sampleto-detector distances $(1.33 \mathrm{~m}, 4 \mathrm{~m}$, and $13.2 \mathrm{~m})$, to access a scattering vector range of approximately 0.001 to $0.5 \AA^{-1}$. The scattered beam was counted on a $2 \mathrm{D}{ }^{3} \mathrm{He}$ detector and radiallyaveraged to produce 1D scattering curves of total intensity against the scattering vector (I vs Q). Data were reduced using Igor Pro and NCNR developed reduction scripts. ${ }^{65}$ Small angle X-ray scattering (SAXS) aLUV data were collected with a Rigaku BioSAXS2000 small angle instrument outfitted with a Pilatus $100 \mathrm{~K}$ detector and a HF007 rotating copper anode (Rigaku Americas, The Woodlands, TX). LUV data were collected at Beamline 12-ID-B of at the Advanced Photon Source, Argonne National Laboratory. aLUV and LUV data were collected at $288 \mathrm{~K}, 303 \mathrm{~K}$ and $318 \mathrm{~K}$. SAXS data were analyzed by the joint refinement of SAXS and SANS data using a symmetric 5-strip model similar to references 2527 .

\section{7 aLUV Assessment}

Asymmetric lipid distributions in aLUVs were quantified from solution ${ }^{1} \mathrm{H}$-NMR spectra measured in the presence of extravesicular paramagnetic lanthanide ions $\operatorname{Pr}^{3+}$. Externally added $\operatorname{Pr}^{3+}$ does not permeate into the vesicle interior on the timescale of the NMR measurement, 22 and therefore interacts only with outer leaflet lipid headgroups to induce a downfield shift of the ESM choline resonance. We note that the ethanolamine of POPE does not contribute to the ${ }^{1} \mathrm{H}$-NMR signal. The observed choline signal is a superposition of shifted and unshifted resonances whose relative areas are proportional to the amount of ESM in the outer and inner leaflets, respectively (Fig. 1, A). ${ }^{22}$ Immediately prior to SANS and NSE measurements aLUVs whose outer leaflets were enriched in ESM (i.e., POPE ${ }^{\text {in }} / \mathrm{ESM}^{\text {out }}$ ), exhibited an unequal area ratio (Fig. 1. upper panel). The ESM mole fraction in the outer and inner leaflet were 0.44 and 0.06 respectively. This aLUV lipid configuration remained over the course of the NSE measurements, as confirmed by NMR post measurement. The observed temporal stability of asymmetry is consistent with recent flip-flop studies of long chained phospholipids. 25

\section{Conflicts of interest}

There are no conflicts to declare.

\section{Acknowledgements}

The authors thank Rob Brinson for NMR support. This work acknowledges support from: the Natural Sciences and Engineering Research Council (NSERC) of Canada [RGPIN-2018-04841] (D.M.); the Ontario Graduate Scholarship (OGS) program (M.H.L.N. and M.D.). M.N. acknowledges funding support of cooperative agreement No. 70NANB15H259 from the National Institute of Standards and Technology, U.S. Department of Commerce. F.A.H. acknowledges support from National Science Foundation (NSF) grant No. MCB-1817929. This material is based upon activities supported by the NSF under Agreement No. DMR-9986442. Access to the Neutron Spin Echo Spectrometer and NGB30 SANS Instrument was provided by the Center for High Resolution Neutron Scattering, a partnership between NIST and the NSF under Agreement No. DMR-1508249. This research used resources of the Advanced Photon Source, a U.S. Department of Energy (DOE) Office of Science User Facility operated for the DOE Office of Science by Argonne National Laboratory under Contract No. DEAC02-06CH11357.

\section{Notes and references}

1 R. M. Bell, L. M. Ballas and R. A. Coleman, J. Lipid Res., 1981, 22, 391-403.

2 T. Pomorski, S. Hrafnsdóttir, P. F. Devaux and G. van Meer, Semin. Cell. Dev. Biol., 2001, 12, 139-148.

3 M. A. Kol, de Kroon, Anton I P M, J. A. Killian and B. de Kruijff, Biochemistry, 2004, 43, 2673-2681.

4 P. F. Devaux and R. Morris, Traffic (Copenhagen, Denmark), 2004, 5, 241-6.

5 D. Marquardt, B. Geier and G. Pabst, Membranes, 2015, 5, 180-196.

6 A. C. Woodka, P. D. Butler, L. Porcar, B. Farago and M. Nagao, Phys. Rev. Lett., 2012, 109, 058102.

7 M. Nagao, E. G. Kelley, R. Ashkar, R. Bradbury and P. D. Butler, The Journal of Physical Chemistry Letters, 2017, 8, 4679-4684.

8 D. Marsh, Chemistry and Physics of Lipids, 2006, 144, 146 159.

9 M. Simunovic and G. A. Voth, Nature Communications, 2015, 6, year.

10 T. Chou, K. S. Kim and G. Oster, Biophysical Journal, 2001, 80, 1075-1087.

11 J. A. Lundbæk, P. Birn, S. E. Tape, G. E. S. Toombes, R. Søgaard, R. E. Koeppe, S. M. Gruner, A. J. Hansen and O. S. Andersen, Molecular Pharmacology, 2005, 68, 680-689.

12 P. I. Kuzmin, S. A. Akimov, Y. A. Chizmadzhev, J. Zimmerberg and F. S. Cohen, Biophysical Journal, 2005, 88, 1120 - 1133.

13 T. Baumgart, S. T. Hess and W. W. Webb, Nature, 2003, 425 821-824.

14 R. Lipowsky, Journal of Biological Physics, 2002, 28, 195-210.

15 F. Jülicher and R. Lipowsky, Phys. Rev. Lett., 1993, 70, 2964 2967. 
16 J. Liu, M. Kaksonen, D. G. Drubin and G. Oster, Proceedings of the National Academy of Sciences, 2006, 103, 10277-10282.

17 E. Sackmann, FEBS Letters, 1994, 346, 3 - 16.

18 Y. Elani, S. Purushothaman, P. J. Booth, J. M. Seddon, N. J. Brooks, R. V. Law and O. Ces, Chem. Commun., 2015, 51, 6976-6979.

19 R. J. Bingham, S. W. Smye and P. D. Olmsted, EPL (Europhysics Letters), 2015, 111, 18004.

20 G. van Meer, D. R. Voelker and G. W. Feigenson, Nature Reviews Molecular Cell Biology, 2008, 9, 112-124.

21 F. A. Heberle, D. Marquardt, M. Doktorova, B. Geier, R. F. Standaert, P. Heftberger, B. Kollmitzer, J. D. Nickels, R. A. Dick, G. W. Feigenson, J. Katsaras, E. London and G. Pabst, Langmuir, 2016, 32, 5195-5200.

22 M. Doktorova, F. A. Heberle, B. Eicher, R. F. Standaert, J. Katsaras, E. London, G. Pabst and D. Marquardt, Nature Protocols, 2018, 13, 2086-2101.

23 B. Eicher, F. A. Heberle, D. Marquardt, G. N. Rechberger, J. Katsaras and G. Pabst, Journal of Applied Crystallography, 2017, 50, 419-429.

24 B. Eicher, D. Marquardt, F. A. Heberle, I. Letofsky-Papst, G. N. Rechberger, M.-S. Appavou, J. Katsaras and G. Pabst, Biophysical Journal, 2018, 114, 146-157.

25 D. Marquardt, F. A. Heberle, T. Miti, B. Eicher, E. London, J. Katsaras and G. Pabst, Langmuir, 2017, 33, 3731-3741.

26 B. Perly, I. C. P. Smith, L. Hughes, G. W. Burton and K. U. Ingold, Biochim. Biophys. Acta, 1985, 819, 131-135.

27 M. Doktorova, F. A. Heberle, D. Marquardt, R. Rusinova, R. L. Sanford, T. A. Peyear, J. Katsaras, G. W. Feigenson, H. Weinstein and O. S. Andersen, Biophysical Journal, 2019, 116, 860 -873 .

28 J.-H. Lee, S.-M. Choi, C. Doe, A. Faraone, P. A. Pincus and S. R. Kline, Physical Review Letters, 2010, 105, 038101.

29 M. Mell, L. H. Moleiro, Y. Hertle, P. Fouquet, R. Schweins, I. Lopez-Montero, T. Hellweg and F. Monroy, The European Physical Journal E, 2013, 75, 75-97.

30 M. Mell, L. H. Moleiro, Y. Hertle, I. Lopez-Montero, F. J. Cao, P. Fouquet, T. Hellweg and F. Monroy, Chemistry and Physics of Lipids, 2015, 185, 61-77.

31 A. G. Zilman and R. Granek, Phys. Rev. Lett., 1996, 77, 47884791.

32 U. Seifert and S. A. Langer, Europhysics Letters, 1993, 23, 7176.

33 M. C. Watson and F. L. H. Brown, Biophysical Journal, 2010, 98, L09-L11.

34 E. G. Kelley, P. D. Butler and M. Nagao, Characterization of Biological Membranes, De Gruyter, Berlin, Boston, 2019, pp. 131-176.

35 J. H. Ipsen, G. Karlström, O. G. Mouritsen, H. Wennerström and M. J. Zuckermann, Biochimica et biophysica acta, 1987, 905, 162-72.

36 N. Kučerka, M.-P. Nieh and J. Katsaras, Biochimica et biophysica acta, 2011, 1808, 2761-71.

37 J. L. Rubenstein, B. A. Smith and H. M. McConnell, Proceed- ings of the National Academy of Sciences, 1979, 76, 15-18.

38 B. Wah, J. M. Breidigan, J. Adams, P. Horbal, S. Garg, L. Porcar and U. Perez-Salas, Langmuir, 2017, 33, 3384-3394.

39 F. A. Heberle and G. W. Feigenson, Cold Spring Harbor Perspectives in Biology, 2011, 3, a004630-a004630.

40 R. Dimova, Advances in Colloid and Interface Science, 2014, 208, 225-234.

41 R. Ashkar, M. Nagao, P. D. Butler, A. C. Woodka, M. K. Sen and T. Koga, Biophysical Journal, 2015, 109, 106-112.

42 E. G. Kelley, P. D. Butler and M. Nagao, Soft Matter, 2019, 15, 2762-2767.

43 Netz and Pincus, Physical review. E, Statistical physics, plasmas, fluids, and related interdisciplinary topics, 1995, 52, 4114-4128.

44 B. Kollmitzer, P. Heftberger, M. Rappolt and G. Pabst, Soft Matter, 2013, 9, 10877.

45 M. Nguyen, M. DiPasquale, B. Rickeard, C. Stanley, E. Kelley and D. Marquardt, Biophysical Journal, 2019, 116, 755-759.

46 S. P. Soni, D. S. LoCascio, Y. Liu, J. A. Williams, R. Bittman, W. Stillwell and S. R. Wassall, Biophysical Journal, 2008, 95, 203-214.

47 S. R. Shaikh, A. C. Dumaual, A. Castillo, D. LoCascio, R. A. Siddiqui, W. Stillwell and S. R. Wassall, Biophysical Journal, 2004, 87, 1752-1766.

48 I. V. Polozov and K. Gawrisch, Biophysical Journal, 2004, 87, 1741-1751.

49 J. R. Silvius, Lipid-protein interactions, 1982, 2, 239-281.

$50 \mathrm{~L} . \mathrm{Wu}$ and X. Jiang, Analytical and Bioanalytical Chemistry, 2016, 408, 2743-2758.

51 L. Wang, P. Quan, S. H. Chen, W. Bu, Y.-F. Li, X. Wu, J. Wu, L. Zhang, Y. Zhao, X. Jiang, B. Lin, R. Zhou and C. Chen, ACS Nano, 2019, 13, 8680-8693.

52 W. Rawicz, K. Olbrich, T. McIntosh, D. Needham and E. Evans, Biophysical Journal, 2000, 79, 328-339.

53 L. R. Arriaga, R. Rodríguez-García, I. López-Montero, B. Farago, T. Hellweg and F. Monroy, The European Physical Journal E, 2010, 31, 105-113.

54 K. Karamdad, R. V. Law, J. M. Seddon, N. J. Brooks and O. Ces, Chemical Communications, 2016, 52, 5277-5280.

55 W. Helfich, Zeitschrift Fur Naturforschug Section A-A Journal of Physics Sciences, 1978, 33, 304-315.

56 N. Dan, P. Pincus and S. A. Safran, Langmuir, 1993, 9, 27682771.

57 S. F. Fenz, T. Bihr, R. Merkel, U. Seifert, K. Sengupta and A.-S. Smith, Nature Physics, 2017, 13, 906-913.

58 G. Chang, R. H. Spencer, A. T. Lee, M. T. Barclay and D. C. Rees, Science (New York, N.Y.), 1998, 282, 2220-6.

59 E. Perozo and D. C. Rees, Current opinion in structural biology, 2003, 13, 432-42.

60 S. Dmitrieff and F. Nédélec, PLOS Computational Biology, 2015, 11, e1004538.

61 P. W. Fowler, J. Hélie, A. Duncan, M. Chavent, H. Kolds $\varnothing$ and M. S. P. Sansom, Soft Matter, 2016, 12, 7792-7803.

62 N. Rosov, S. Rathgeber and M. Monkenbusch, Scattering from 
Polymers, American Chemical Society, 1999, vol. 739, ch. 7, pp. 103-116.

63 R. Azuah, L. Kneller, Y. Qiu, P. Tregenna-Piggott, C. Brown, J. Copley and D. RM, J Res Natl Inst Stand Technol., 2009, 114, 347-352.
64 C. J. Glinka, J. G. Barker, B. Hammouda, S. Krueger, J. J. Moyer and W. J. Orts, Journal of Applied Crystallography, 1998, 31, 430-445.

65 S. R. Kline, Journal of Applied Crystallography, 2006, 39, 895900. 